\title{
Padrões de distribuição geográfica das espécies de Chamaecrista sect. Chamaecrista ser. Coriaceae (Benth.) H. S. Irwin \& Barneby, Leguminosae - Caesalpinioideae ${ }^{1}$
}

\author{
JULIANA GASTALDELLO RANDO ${ }^{2,3}$ e JOSÉ RUBENS PIRANI ${ }^{2}$
}

(recebido: 12 de maio de 2010; aceito: 05 de setembro de 2011)

\begin{abstract}
Patterns of geographic distribution of the species of Chamaecrista sect. Chamaecrista ser. Coriaceae (Benth.) H. S. Irwin \& Barneby Leguminosae - Caesalpinioideae). An analysis of the geographic distribution of the 27 taxa belonging to 20 species of Chamaecrista sect. Chamaecrista ser. Coriaceae was carried out, based on maps depicting the known occurrences of the taxa. One species occurs in the Caribbean region, C. caribaea (Northr.) Britton, whereas the others are distributed in a main center of diversity, the Espinhaço Range, presenting two main patterns: 1) a continuous to disjunct occurrence through the Espinhaço Range and adjacent or disjunct areas, and 2) an endemic distribution in the southern region of that mountain range. Among the disjunctions, three species are noteworthy: one occurring in the Espinhaço and elevations of the "Arco de Brasília" westwards Goiás (C. cardiostegia H. S. Irwin \& Barneby), other in the Espinhaço and in Espírito Santo State (C. mucronata (C. Sprengel) H. S. Irwin \& Barneby), and a third one centered in the Espinhaço and in the Guyana Shield (C. roraimae (Benth.) Gleason). The distribution patterns are discussed, mainly as compared to data from other groups of angiosperms, and to a previous analysis of five species belonging to $C$. ser. Coriaceae. The results show a concentration of endemic species in highlands in the Cerrado Domain. They give additional support to the high degree of endemism already documented for other groups in the region. This analysis is an initial step allowing further studies to understand the processes responsible for the diversification of the group in the cerrado, specially in elevated areas.
\end{abstract}

Key words - biogeography, endemism, Espinhaço Range, legumes

RESUMO - (Padrões de distribuição geográfica das espécies de Chamaecrista sect. Chamaecrista ser. Coriaceae (Benth.) H. S. Irwin \& Barneby, Leguminosae - Caesalpinioideae). Uma análise de distribuição geográfica de Chamaecrista sect. Chamaecrista ser. Coriaceae foi realizada com base em mapas atualizados plotando as ocorrências conhecidas de 27 táxons (20 espécies) da série. Apenas uma espécie ocorre na região do Caribe, nas Bahamas, C. caribaea (Northr.) Britton, enquanto todas as demais estão presentes no principal centro de diversidade, a Cadeia do Espinhaço, exibindo dois padrões principais: 1) ocorrência contínua ou disjunta ao longo da Cadeia do Espinhaço e áreas adjacentes ou disjuntas e 2) espécies endêmicas da porção sul dessa formação montanhosa. Entre as disjunções, destacam-se três espécies, uma ocorrendo no Espinhaço e em serras do "Arco de Brasília” até Goiás (C. cardiostegia H. S. Irwin \& Barneby), uma no Espinhaço e no Espírito Santo (C. mucronata (C. Sprengel) H. S. Irwin \& Barneby) e outra centrada no Espinhaço e no Planalto das Guianas (C. roraimae (Benth.) Gleason). Os padrões de distribuição geográfica são discutidos com base em dados obtidos sobre outros grupos de angiospermas e uma análise realizada anteriormente com cinco espécies da série. Os resultados demonstram uma concentração de espécies restrita a regiões de altitude situadas no Domínio do Cerrado, corroborando o alto grau de endemismo já documentado em outros grupos na região. Essa análise constitui um primeiro passo para possibilitar estudos visando à compreensão dos processos responsáveis pela diversificação do grupo no cerrado, sobretudo em áreas elevadas.

Palavras-chave - biogeografia, Cadeia do Espinhaço, endemismo, leguminosas

\section{Introdução}

Chamaecrista Moench inclui cerca de 330 espécies, distribuídas principalmente na América Tropical, raramente África, Ásia e Austrália, com poucas espécies atingindo áreas temperadas (Irwin \& Barneby 1982,

1. Parte da dissertação de mestrado da primeira autora, Programa de Pós-Graduação em Botânica da Universidade de São Paulo, SP, Brasil.

2. Universidade de São Paulo, Instituto de Biociências, Departamento de Botânica, Rua do Matão, Travessa 14, 321, 05508-900 São Paulo, SP, Brasil.

3. Autor para correspondência: ju_rando@hotmail.com
Lewis 2005). No Brasil, ocorrem cerca de 230 espécies. Dessas, 200 estão concentradas na região do Planalto Central Brasileiro, nos Estados de Bahia, Goiás, Minas Gerais e Tocantins (Irwin \& Barneby 1982, Lewis 1987). Segundo diversos autores (Irwin \& Barneby 1982, Lewis 1987, Harley 1988, Conceição et al. 2001, Souza \& Bortoluzzi 2010) os Estados da Bahia e de Minas Gerais, com 105 espécies restritas a eles, são os principais centros de diversidade do grupo, sendo o cerrado e os campos rupestres os locais de destaque por apresentarem diversas espécies endêmicas.

O gênero Chamaecrista pertence à tribo Cassieae e à subtribo Cassiinae, juntamente com os gêneros 
Cassia L. e Senna Mill., e é atualmente reconhecido como monofilético (Conceição et al. 2009). Os caracteres diagnósticos do gênero são: pedicelo com duas bractéolas situadas próximo ou acima da metade de seu comprimento total; androceu actinomorfo com anteras pubescentes ao longo da suturas; fruto elasticamente deiscente; nectários extrafloral concâvo (Irwin \& Barneby 1977, 1982, Conceição et al. 2009). Irwin \& Barneby (1982) reconheceram seis seções no gênero: Chamaecrista sect. Absus, C. sect. Apoucouita, C. sect. Caliciopsis, C. sect. Chamaecrista, C. sect. Grimaldia e C. sect. Xerocalyx. O reconhecimento dessas seções é baseado na combinação de características como padrão de inflorescência, presença ou ausência de tricomas glandulares e padrões de nervuras das sépalas e folíolos. Considerando somente as Américas, ocorrem cerca de 270 espécies (tabela 1), sendo $C$. sect. Absus a mais representativa, com cerca de 170 espécies, facilmente reconhecida por apresentar inflorescência racemosa terminal e tricomas glandulares nas folhas, ramos ou apenas nas inflorescências. Chamaecrista sect. Absus tem grande diversidade nos Estados da Bahia, Goiás e Minas Gerais e pouca ou nenhuma ocorrência nos Estados do Sul do Brasil (Irwin
\& Barneby 1978, Lewis 1987, Camargo \& Miotto 2004). Chamaecrista sect. Apoucouita, com cerca de 19 espécies e representada por diversos indivíduos arbóreos e com inflorescência racemosa cauliflora, está concentrada na região amazônica exibindo disjunção na Bahia, sendo apenas uma espécie, C. ensiformis (Vell.) H. S. Irwin \& Barneby, amplamente distribuída em todas regiões brasileiras, exceto no Sul (Irwin \& Barneby 1977, 1982). As seções menos representativas $C$. sect. Caliciopsis $(2$ spp.) e $C$. sect. Xerocalyx (3 spp.) apresentam distribuição do México até a Argentina, quanto que $C$. sect. Grimaldia (1 sp.) com distribuição pantropical. (Irwin \& Barneby 1978, 1982). A segunda seção mais representativa é $C$. sect. Chamaecrista, que inclui geralmente as plantas subarbustivas a arbustivas, dotadas de inflorescências reduzidas a fascículos axilares, com cerca de 76 espécies distribuídas em seis séries: ser. Bauhinianae, ser. Chamaecrista, ser. Coriaceae, ser. Flexuosae, ser. Greggianae e ser. Prostratae. Essa seção é representada tanto por espécies amplamente distribuídas, como $C$. flexuosa (L.) Greene, C. nictitans (L.) Moench e $C$. rotundifolia (Pers.) Greene, quanto por outras endêmicas ou raras, como várias espécies da ser. Coriaceae.

Tabela 1. Classificação das espécies de Chamaecrista ocorrentes nas Américas, baseada em Irwin \& Barneby (1982).

Table 1. Classification of the species of Chamaecrista with occurrence in the Americas, based on Irwin \& Barneby (1982).

\begin{tabular}{|c|c|c|c|c|c|c|c|}
\hline $\begin{array}{c}\text { Seção e número } \\
\text { de espécies }\end{array}$ & $\begin{array}{l}\text { Apoucouita } \\
\text { (19 spp.) }\end{array}$ & \multicolumn{2}{|c|}{$\begin{array}{c}\text { Absus } \\
\text { (ca. } 170 \text { spp.) }\end{array}$} & $\begin{array}{l}\text { Grimaldia } \\
\text { (1 sp.) }\end{array}$ & $\begin{array}{c}\text { Chamaecrista } \\
\text { (ca. } 76 \text { spp.) }\end{array}$ & $\begin{array}{l}\text { Caliciopsis } \\
\text { (2 spp.) }\end{array}$ & $\begin{array}{c}\text { Xerocalyx } \\
\text { (3 spp.) }\end{array}$ \\
\hline Subseção & & \multicolumn{2}{|c|}{$\begin{array}{l}\text { Absus } \\
\text { Adenophyllum } \\
\text { Baseophyllum } \\
\text { Otophyllum }\end{array}$} & & & & \\
\hline Série & $\begin{array}{l}\text { Apoucouita } \\
\text { Pteridophyllae }\end{array}$ & $\begin{array}{l}\text { Absoideae } \\
\text { Adenophyllae } \\
\text { Andromedeae } \\
\text { Atroglandulosae } \\
\text { Bracteolatae } \\
\text { Catharticae } \\
\text { Confertae } \\
\text { Ericifoliae } \\
\text { Geminatae } \\
\text { Glutinosae } \\
\text { Gracillimae } \\
\text { Hassleranae } \\
\text { Hedysaroides } \\
\text { Incanae } \\
\text { Incurvatae }\end{array}$ & $\begin{array}{l}\text { Lucidae } \\
\text { Lomatopodae } \\
\text { Microphyllae } \\
\text { Nigricantes } \\
\text { Ochnaceae } \\
\text { Oligospermae } \\
\text { Paniculatae } \\
\text { Pinifoliae } \\
\text { Rigidulae } \\
\text { Secundae } \\
\text { Setosae } \\
\text { Spinulosae } \\
\text { Strictifoliae } \\
\text { Trachycarpae } \\
\text { Unijugae } \\
\text { Ursinae }\end{array}$ & & $\begin{array}{l}\text { Chamaecrista } \\
\text { Coriaceae } \\
\text { Bauhinianae } \\
\text { Flexuosae } \\
\text { Greggianae } \\
\text { Prostratae }\end{array}$ & & \\
\hline
\end{tabular}


Chamaecrista ser. Coriaceae apresenta 28 táxons (20 espécies e 8 variedades) e pode ser caracterizada por apresentar espécies subarbustivas a arbustivas com xilopódio, que permite a rebrota de novos ramos depois do fogo, e nectário extrafloral sempre presente (Irwin \& Barneby 1982). Recentemente, Conceição et al. (2009) realizaram um estudo filogenético de Chamaecrista, baseado nos marcadores moleculares trnL-F e ITS. Nesse trabalho a seção Chamaecrista mostrou-se parafilética, enquanto as séries Chamaecrista e Coriaceae apareceram como monofiléticas, embora com pequena amostragem. As espécies de Chamaecrista ser. Coriaceae parecem estar distribuídas principalmente em áreas altas do cerrado (nos campos rupestres), com um alto grau de endemismos e interessantes disjunções. Essas características de distribuição tornam a série um grupo adequado para realização de estudos biogeográficos.

O presente trabalho visou mapear a distribuição geográfica das espécies de Chamaecrista sect. Chamaecrista ser. Coriaceae e discutir os padrões de distribuição obtidos, comparando com outros táxons que exibem padrões semelhantes. O conhecimento da distribuição geográfica desta série permitirá estudos futuros visando à compreensão dos processos responsáveis pela diversificação do grupo em áreas de cerrado.

\section{Materiais e métodos}

Mapas de distribuição geográfica das 20 espécies de Chamaecrista sect. Chamaecrista ser. Coriaceae, totalizando
27 táxons ao se considerarem as variedades aqui analisadas (tabela 2), foram realizados a partir do Mapa Base Global GIS no programa ArcView GIS 3.2 (Esri 1999). Foram plotadas as localidades de ocorrência comprovada baseando-se em parte do tratamento taxonômico realizado em Rando (2009), no trabalho de Irwin \& Barneby (1982), no checklist de Funk et al. (2007), Boggan et al. (1997) e no exame das coleções dos seguintes herbários, de acordo com Thiers (2009): ALCB, BHCB, ESA, F, GH, HRCB, HUEFS, K, MBM, NY, OUPR, RB, SP, SPF, UB, UEC, UFG, US. No caso de $C$. potentilla, que apresenta duas variedades $(C$. potentilla var. potentilla e C. potentilla var. specuum H. S. Irwin \& Barneby), foi analisada a distribuição somente ao nível de espécie. Essa decisão foi tomada devido à observação de sobreposição dos caracteres morfológicos utilizados na distinção dessas variedades.

As coordenadas geográficas utilizadas para a plotagem dos mapas de distribuição foram obtidas diretamente no campo com GPS, das etiquetas das exsicatas ou por meio de índices de localidades eletrônicos como o IBGE (http:// www.ibge.org.br), CRIA - Species link (http://splink.cria. org.br/geoloc) e o Global Gazetteer (http://fallingrain.com/ world). Os critérios utilizados para estabelecer os padrões de distribuição dos táxons incluíram análise dos limites norte e sul de ocorrência e da amplitude de distribuição no Brasil e em áreas extrabrasileiras. Adotou-se definição de domínios vegetacionais do Brasil proposta por Ab'Saber (1967), discutida e atualizada por Joly et al. (1999); adotouse o sistema de Morrone (2001) para a região do Caribe. Para uma geografia atualizada do Brasil utilizou-se Ross (1996). Para parte da análise e discussão dos resultados, foi realizada uma busca na literatura disponível de grupos taxonômicos exibindo padrões semelhantes aos encontrados, incluindo

Tabela 2. Espécies de Chamaecrista sect. Chamaecrista ser. Coriaceae, domínios fitogeográficos em que aparecem $(\mathrm{CE}=\mathrm{Cerrado} ; \mathrm{CAA}=$ Caatinga; $\mathrm{MA}=$ Mata Atlântica; $\mathrm{FSC}=$ Florestas Secas de Coníferas $)$, estados ou distritos ou províncias onde podem ser encontradas e o padrão de distribuição aqui definido (ver texto).

Table 2. Species of Chamaecrista sect. Chamaecrista ser. Coriaceae, their phytogeographic domains $(\mathrm{CE}=$ Cerrado; $\mathrm{CAA}=$ Caating $; \mathrm{MA}=$ Atlantic Rain Forest $; \mathrm{FSC}=$ Dry and Conifers Forests $)$, the states or districts or province where they can be found and pattern of distribution defined (see text).

\begin{tabular}{lllll}
\hline \multicolumn{1}{c}{ Espécies } & Domínios & \multicolumn{1}{c}{ País } & Estados ou Distritos ou Províncias & $\begin{array}{c}\text { Padrão } \\
\text { distr. }\end{array}$ \\
\hline C. anceps (Benth.) H. S. Irwin \& Barneby & CE & Brasil & Minas Gerais & 2.1 \\
C. aristata (Benth.) H. S. Irwin \& Barneby & CE & Brasil & Minas Gerais & 2.1 \\
C. burchellii (Benth.) H. S. Irwin \& Barneby & CE & Brasil & Goiás & 4 \\
C. cardiostegia H. S. Irwin \& Barneby & CE & Brasil & Goiás, Minas Gerais, Paraná & 3.1 \\
C. caribaea (Northr.) Britton var. caribaea & FSC & Bahamas & Distritos de Andros e Cat Island & 5 \\
C. caribaea var. lucayana (Britton) H. S. Irwin & FSC & Bahamas & Distritos de Cat Island, Conception & 5 \\
\& Barneby & & & Island, Exuma, San Salvador e & Rum Cay
\end{tabular}


continuação

\begin{tabular}{|c|c|c|c|c|}
\hline Espécies & Domínios & País & Estados ou Distritos ou Províncias & $\begin{array}{c}\text { Padrão } \\
\text { distr. }\end{array}$ \\
\hline $\begin{array}{l}\text { C. caribaea var. inaguensis (Britton) H. S. Irwin } \\
\text { \& Barneby }\end{array}$ & FSC & $\begin{array}{l}\text { Bahamas, } \\
\text { Haiti }\end{array}$ & $\begin{array}{l}\text { Distritos de Turks \& Caicos Islands e } \\
\text { Inagua }\end{array}$ & 5 \\
\hline $\begin{array}{l}\text { C. choriophylla (Vogel) H. S. Irwin \& Barneby } \\
\text { var. choriophylla }\end{array}$ & $\mathrm{CE}$ & Brasil & Minas Gerais & 2.1 \\
\hline $\begin{array}{l}\text { C. choriophylla var. latifolia (Benth.) H. S. Irwin } \\
\text { \& Barneby }\end{array}$ & $\mathrm{CE}$ & Brasil & Minas Gerais & 2.1 \\
\hline $\begin{array}{l}\text { C. choriophylla var. rossicorum H. S. Irwin \& } \\
\text { Barneby }\end{array}$ & $\mathrm{CE}$ & Brasil & Minas Gerais & 3.1 \\
\hline C. cinerascens (Vogel) H. S. Irwin \& Barneby & $\mathrm{CE}$ & Brasil & Minas Gerais & 2.1 \\
\hline C. distichoclada (Benth.) H. S. Irwin \& Barneby & $\mathrm{CE}$ & Brasil & Minas Gerais & 2.1 \\
\hline C. lagotois H. S. Irwin \& Barneby & $\mathrm{CE}$ & Brasil & Minas Gerais & 2.2 \\
\hline C. mucronata (Spreng.) H. S. Irwin \& Barneby & CE, MA & Brasil & Bahia, Espírito Santo, Minas Gerais & 3.2 \\
\hline C. multinervea (Benth.) H. S. Irwin \& Barneby & $\mathrm{CE}$ & Brasil & Bahia, Minas Gerais & 1 \\
\hline C. olesiphylla (Vogel) H. S. Irwin \& Barneby & $\mathrm{CE}$ & Brasil & Minas Gerais & 2.1 \\
\hline C. papillata H. S. Irwin \& Barneby & $\mathrm{CE}$ & Brasil & Bahia, Minas Gerais & 1 \\
\hline C. potentilla (Benth.) H. S. Irwin \& Barneby & $\mathrm{CE}$ & Brasil & Minas Gerais & 3.1 \\
\hline C. roraimae (Benth.) Gleason & $\mathrm{CAA}, \mathrm{CE}$ & $\begin{array}{l}\text { Brasil, } \\
\text { Guiana e } \\
\text { Venezuela }\end{array}$ & $\begin{array}{l}\text { Bahia, Minas Gerais (fora do } \\
\text { Espinhaço), Roraima e Províncias } \\
\text { de Bolívar e Potaro-Siparuni. }\end{array}$ & 3.3 \\
\hline $\begin{array}{l}\text { C. rotundata var. grandistipula (Vogel) } \\
\text { H. S. Irwin \& Barneby }\end{array}$ & $\mathrm{CE}$ & Brasil & Minas Gerais & 2.1 \\
\hline $\begin{array}{l}\text { C. rotundata var. interstes H. S. Irwin \& } \\
\text { Barneby }\end{array}$ & $\mathrm{CE}$ & Brasil & Minas Gerais & 3.1 \\
\hline $\begin{array}{l}\text { C. rotundata (Vogel) H. S. Irwin \& Barneby var. } \\
\text { rotundata }\end{array}$ & $\mathrm{CE}$ & Brasil & Minas Gerais & 2.1 \\
\hline C. simplifacta H. S. Irwin \& Barneby & $\mathrm{CE}$ & Brasil & Minas Gerais & 2.2 \\
\hline $\begin{array}{l}\text { C. tragacanthoides var. rasa H. S. Irwin \& } \\
\text { Barneby }\end{array}$ & $\mathrm{CE}$ & Brasil & Minas Gerais & 2.1 \\
\hline $\begin{array}{l}\text { C. tragacanthoides (Benth.) H. S. Irwin \& } \\
\text { Barneby var. tragacanthoides }\end{array}$ & $\mathrm{CE}$ & Brasil & Minas Gerais & 2.1 \\
\hline C. ulmea H. S. Irwin \& Barneby & $\mathrm{CE}$ & Brasil & Minas Gerais & 2.2 \\
\hline C. venulosa (Benth.) H. S. Irwin \& Barneby & $\mathrm{CE}$ & Brasil & Bahia, Minas Gerais & 1 \\
\hline
\end{tabular}

uma análise prévia com cinco espécies de Chamaecrista ser. Coriaceae realizada por Giulietti \& Pirani (1988). Os nomes completos dos binômios e trinômios, com autoridades, são apresentados na tabela 2 .

\section{Resultados e discussão}

Dos 27 táxons de Chamaecrista sect. Chamaecrista ser. Coriaceae, 19 espécies e 24 táxons podem ser agrupados em um dos domínios fitogeográficos que mais contribuem na formação das paisagens naturais do Brasil, o Cerrado (tabela 2). A única espécie que não foi enquadrada nessa formação é $C$. caribaea, que apresenta a distribuição restrita à região do Caribe.
No domínio do Cerrado, as espécies de Chamaecrista ser. Coriaceae se encontram predominantemente em terrenos elevados, acima de $900 \mathrm{~m}$ de altitude, apresentando um elevado grau de endemismo nos campos rupestres. Nessas regiões merece destaque a Cadeia do Espinhaço (18 espécies, 24 táxons), principalmente a região localizada no Estado de Minas Gerais, onde ocorrem 17 espécies da série (23 táxons), sendo 12 espécies (17 táxons) exclusivas da parte mineira.

A distribuição da série ao longo do Espinhaço apresenta seis táxons disjuntos: Chamaecrista mucronata, que ocorre no leste do Espírito Santo; C. roraimae disjunta nas elevadas altitudes localizadas no monte Roraima; C. choriophylla var. rossicorum, C. potentilla e 
C. rotundata var. interstes em serras do "Arco de Brasília", que incluem as elevações da região de Furnas e da Serra da Canastra no leste de Minas Gerais e as da região da Serra dos Cristais, nos limites de Minas Gerais e Goiás, com destaque para C. cardiostegia que se estende até outras serras de Goiás. Apenas cinco espécies ocorrem por quase toda extensão da Cadeia do Espinhaço, com registros desde o setor sul (Minas Gerais) até o setor norte (Chapada Diamantina, na Bahia), porém nenhum endemismo foi observado no setor baiano. A única espécie endêmica de outras formações rupestres fora da Cadeia do Espinhaço é C. burchellii, que ocorre exclusivamente no estado de Goiás, a oeste da Chapada dos Veadeiros.

$\mathrm{Na}$ porção mineira da Cadeia do Espinhaço, aparentemente a série sofreu uma diversificação significativa, como sugerido por Giulietti \& Pirani (1988) para Chamaecrista e outros gêneros, como Leiothrix (Eriocaulaceae), Pseudotrimezia (Iridaceae) e Senna (Leguminosae). Barbacenia (Velloziaceae), Eriope (Lamiaceae) e Schefflera (Araliaceae) também apresentam intensa diversificação na mesma região (Mello-Silva 1995, Harley 1988, Fiaschi \& Pirani 2008). O elevado grau de endemismo ao longo da Cadeia do Espinhaço (Minas Gerais e Bahia) é situação comum, observada em diversos grupos (Simon \& Proença 2000, Rapini et al. 2001, 2002, 2009, Fiaschi \& Pirani 2008, 2009, Giulietti et al. 2009) e reconhecida por diversos autores (Joly 1970, Giulietti \& Pirani 1988, Prance 1994, Harley 1995, Giulietti et al. 1997, 2009). A área de campo rupestre localizada na Chapada do Veadeiros, em Goiás, é uma outra região de destaque por apresentar muitas espécies endêmicas. A quantidade de endemismos posicionou Goiás como terceiro estado com maior número de espécies raras de angiospermas do Brasil (202), seguindo os Estados de Minas Gerais (550) e Bahia (484) (Giulietti et al. 2009). A expressiva representatividade de espécies raras nesses três estados é atribuída a grande quantidade de endemismos pontuais nas regiões de campos rupestres ali existentes (Giulietti et al. 2009).

Padrões de distribuição geográfica de Chamaecrista sect. Chamaecrista ser. Coriaceae - A presente análise das espécies de $C$. sect. $C$. ser. Coriaceae permitiu o estabelecimento dos seguintes padrões de distribuição geográfica, organizados de acordo com a amplitude de distribuição em termos de área de cada espécie: 1) distribuição ao longo da Cadeia do Espinhaço; 2) distribuição restrita ao setor mineiro (sul) da Cadeia do Espinhaço: 2.1) espécies que ocorrem em mais de uma serra, 2.2) espécies endêmicas de uma única serra; 3) distribuição na Cadeia do Espinhaço adjacentes até áreas disjuntas: 3.1) distribuição concentrada em serras do Espinhaço em áreas adjacentes até disjuntas no Planalto Central; 3.2) distribuição em serras do Espinhaço e em uma área disjunta no Espírito Santo; 3.3) distribuição centrada em serras do Espinhaço e adjacências com uma área disjunta no norte da América do Sul; 4) distribuição restrita a outra região no Brasil; 5) distribuição restrita à região extrabrasileira.

1. Distribuição ao longo da Cadeia do Espinhaço As espécies incluídas nessa categoria apresentam distribuição exclusiva mais ou menos contínua do sul dessa formação montanhosa até o norte, ou claramente disjunta entre os seus setores baiano e mineiro. Esse padrão foi verificado em C. multinervea (figura 1A), C. papillata (figura 1B) e C. venulosa (figura 1A). Chamaecrista multinervea é conhecida apenas por três registros: o holótipo, proveniente da região de Diamantina (Minas Gerais), e dois registros mais recentes na Chapada Diamantina, Bahia (Mucugê e Rio de Contas), porém a sua grande semelhança morfológica com C. mucronata deixa em questão se realmente é uma espécie distinta ou apenas uma variação desta. Chamaecrista papillata é frequente na região da Serra do Cipó, enquanto na Bahia há poucas coletas. É, também, proximamente relacionada com C. mucronata, sendo distintas, principalmente, pela presença de papilas na epiderme dos folíolos em C. papillata. Porém, essa distinção é geralmente dificultada pelo fato de os estômatos de C. mucronata apresentarem as células subsidiárias ligeiramente projetadas sobre as célulasguarda, assemelhando-se a papilas quando observadas somente sob estereomicroscópio (Francino 2006). Por outro lado, o hábito das duas espécies parece ser distintivo: pelo menos nos indivíduos observados em campo, C. mucronata é um arbusto ereto, enquanto $C$. papillata é prostrada. A falta dessa informação nas exsicatas torna impraticável a identificação segura de material dessas espécies, devido ao grande número de indivíduos com características foliares intermediárias. Estudos taxonômicos acurados são necessários neste complexo, que envolve também C. multinervea e, provavelmente, as informações de distribuição geográficas ou, se possível, de natureza filogeográfica prestarão subsídio importante nessas análises. Chamaecrista venulosa é comum tanto na região sul quanto na região norte da Cadeia do Espinhaço, sendo conhecida de muitas coletas na Serra do Cipó, algumas no Planalto de Diamantina e outras na Chapada Diamantina (Pico das Almas, Rio 

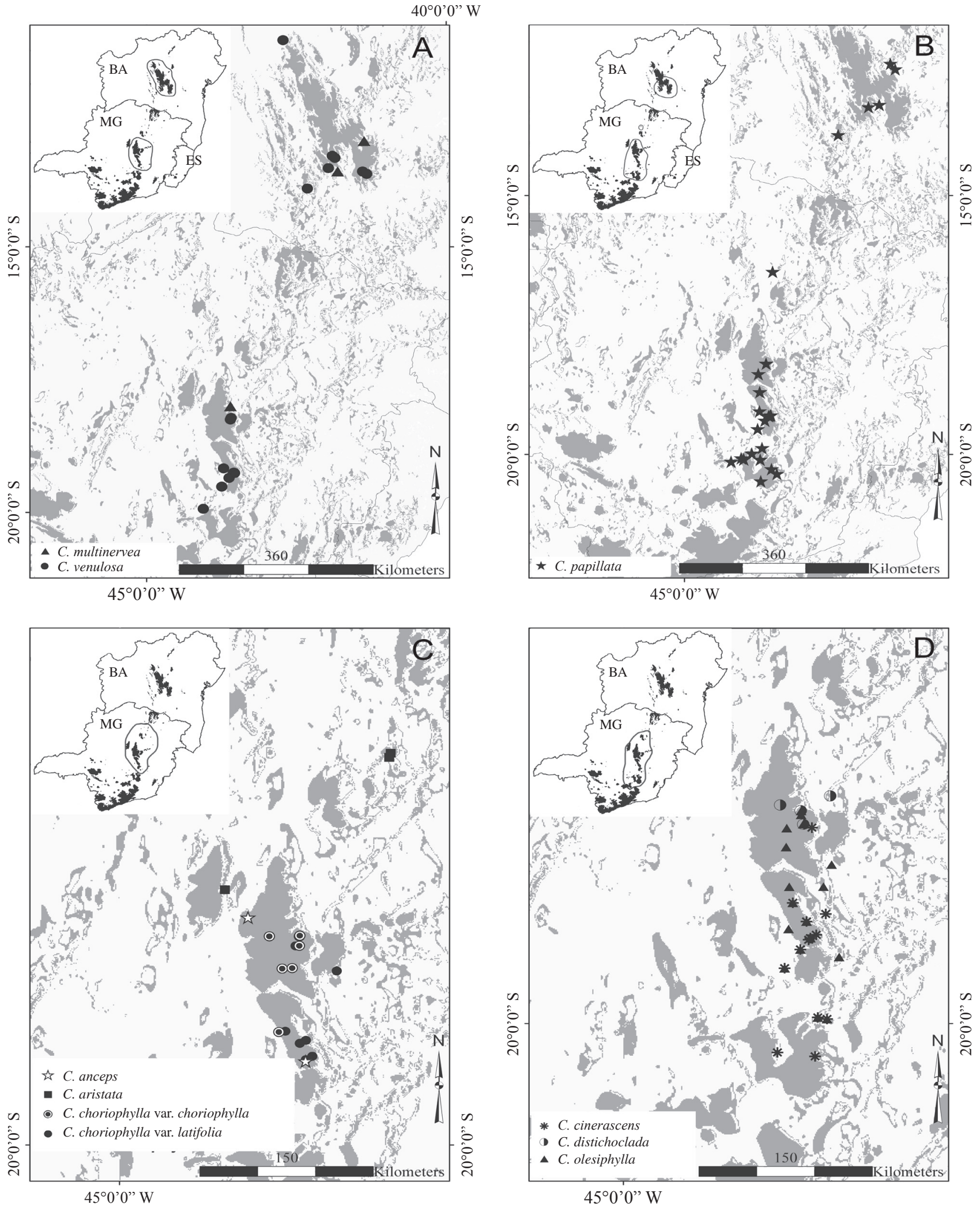

Figura 1. A-B. Distribuição ao longo da Cadeia do Espinhaço. C-D. Distribuição restrita ao setor mineiro (sul) do Espinhaço: espécies que ocorrem em mais de uma serra ( $\mathrm{BA}=$ Bahia; $\mathrm{MG}=$ Minas Gerais).

Figure 1. A-B. Distribution along the Espinhaço Range. C-D. Distribution restricted to southern portion of the Espinhaço: species that occur in more than one "serra" (BA = Bahia; $M G=$ Minas Gerais). 
de Contas). Observam-se variações morfológicas nas populações dos dois setores, sendo as plantas da Bahia mais pilosas, com tricomas áureos e folíolos geralmente maiores. Esses três táxons se enquadram no padrão de espécies que ocorrem em mais de uma serra do Espinhaço, ocupando áreas tanto na Bahia quanto em Minas Gerais, proposto por Giulietti \& Pirani (1988).

2. Distribuição restrita ao setor sul (mineiro) da Cadeia do Espinhaço - Padrão aqui ilustrado por Chamaecrista anceps, $C$. aristata, $C$. choriophylla var. choriophylla, $C$. choriophylla var. latifolia (figura 1C), C. cinerascens, $C$. distichoclada, C. olesiphylla (figura 1D), C. rotundata var. grandistipula e C. rotundata var. rotundata (figura 2A), C. tragacanthoides var. tragacanthoides e $C$. tragacanthoides var. rasa (figura 2B), C. lagotois, $C$. simplifacta e C. ulmea (figura 2C), todas endêmicas da porção sul da Cadeia do Espinhaço, em Minas Gerais, principalmente nas regiões da Serra do Cipó, Diamantina e Grão-Mogol. A maioria dessas espécies apresenta características morfológicas semelhantes e marcantes, como o número de folíolos ou o tipo de estípula, diferentes das espécies que apresentam ampla distribuição, embora haja algumas exceções. As espécies incluídas nessa categoria podem exibir dois padrões já propostos por Giulietti \& Pirani (1988): espécies que ocorrem em mais de uma serra (2.1) e espécies endêmicas de uma única serra (2.2).

2.1. Espécies que ocorrem em mais de uma serra Chamaecrista aristata era considerada endêmica da Serra de Grão-Mogol (Irwin \& Barneby 1982, Queiroz et al. 2004), mas agora é conhecida de coletas adicionais de uma região a sudoeste, a Serra do Cabral (Município de Joaquim Felício). É uma espécie considerada rara (Giulietti et al. 2009). Chamaecrista anceps é conhecida de apenas três coletas: o holótipo e um outro material, ambos da região de Diamantina, e um espécime mais recente oriundo da Serra do Cipó. Há possibilidade de que $C$. anceps seja uma forma maior de $C$. cardiostegia, necessitando de mais estudos taxonômicos. Chamaecrista distichoclada e C. olesiphylla têm sido coletadas, respectivamente, no Planalto de Diamantina e da Serra do Cipó até Diamantina. Chamaecrista olesiphylla é comumente encontrada no Município de Congonhas do Norte, região nordeste da Serra do Cipó. Chamaecrista tragacanthoides é restrita a uma região de Diamantina, sendo que apenas a var. rasa apresenta uma distribuição um pouco mais ampla, presente mais ao norte em Bocaiúva, Montes Claros e Francisco Sá. Duas variedades de C. rotundata, var. grandistipula e var. rotundata, distribuem-se do Planalto de Diamantina, onde são pouco comuns, até o extremo sul do Espinhaço, na região de Lavras, São José Del Rey e Ouro Preto. Em particular, C. rotundata var. grandistipula apresenta muitas coletas no Parque Estadual do Pico do Itacolomi, e um recente registro na região noroeste da Serra do Cipó (Santana do Pirapama). Diferentemente das outras duas variedades, C. rotundata var. interstes é conhecida de muitas coletas na região de Diamantina e de um registro disjunto na região do Arco de Brasília (Ross 1996), enquadrando-se por isso no padrão 3.1. Chamaecrista choriophylla é uma das espécies mais comuns da série. Suas variedades apresentam distribuições distintas: a var. latifolia é frequente apenas no setor sul da Serra do Cipó (Jaboticatubas e Santana do Riacho), enquanto a var. choriophylla, que era conhecida de coletas na região de Diamantina e Gouveia, teve recentemente diversas populações encontradas no setor noroeste da Serra do Cipó (Santana do Pirapama). Já C. choriophylla var. rossicorum tem distribuição mais ampla e teve que ser enquadrada no padrão 3.1 (ver abaixo).

2.2. Espécies endêmicas de uma única serra Chamaecrista ulmea é endêmica da Serra de Grão-Mogol (Irwin \& Barneby 1982, Queiroz et al. 2004), considerada espécie rara (Giulietti et al. 2009), tendo apenas seis registros conhecidos. Chamaecrista lagotois é endêmica da Serra do Cipó e na região são conhecidas apenas três populações. Chamaecrista simplifacta, também considerada rara (Giulietti et al. 2009), tem escassa representação nos herbários, concentrada na região de Diamantina, principalmente na estrada que leva a Conselheiro Mata.

3. Distribuição em Serras do Espinhaço e áreas adjacentes até disjuntas

3.1. Distribuição na Cadeia do Espinhaço e áreas adjacentes até disjuntas no Planalto Central - Neste padrão estão inclusas as áreas de cerrado e/ou serras do Arco de Brasília, que se estendem do trecho a sudoeste do Espinhaço até sudeste de Goiás. Esse padrão foi verificado em $C$. cardiostegia, $C$. potentilla, $C$. choriophylla var. rossicorum e $C$. rotundata var. interstes (figura 2D), devendo ser ressaltado que as demais variedades destas duas últimas espécies exibem o padrão 2.1. Embora a maior parte das coletas de C. choriophylla var. rossicorum provenham da região centro-sul do setor mineiro (Diamantina e Serra do Cipó), existe um registro em Delfinópolis, próximo à Serra da Canastra. Situação semelhante foi observada para $C$. rotundata var. interstes que apresenta muitas coletas na região de Diamantina, mas com uma coleta disjunta na região das serras do 

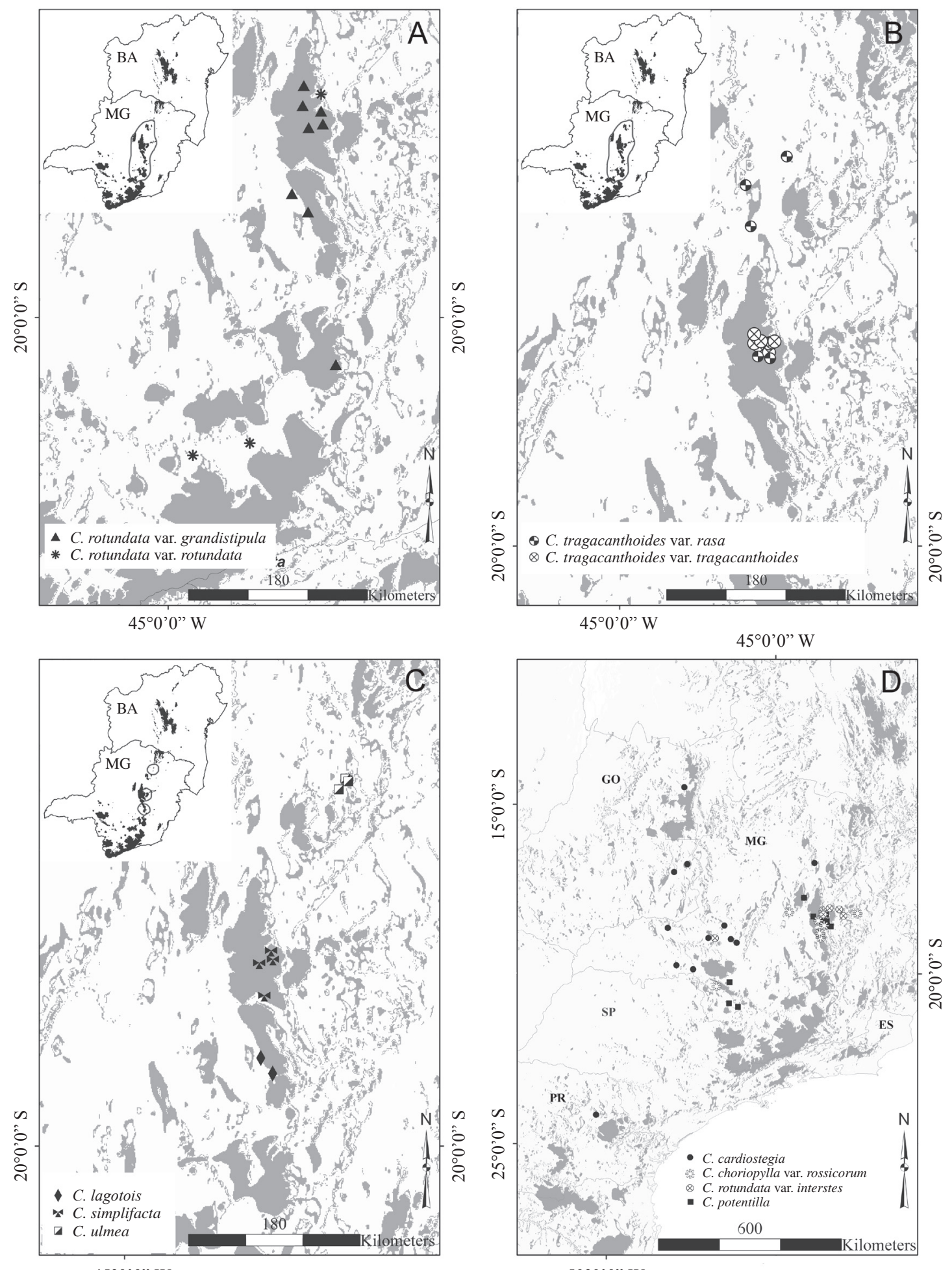

Figura 2. A-C. Distribuição restrita ao setor mineiro (sul) da Cadeia do Espinhaço: A-B. Espécies que ocorrem em mais de uma serra. C. Espécies endêmicas de uma única serra. D. Distribuição na Cadeia do Espinhaço e áreas adjacentes até disjuntas no Planalto Central (BA = Bahia; ES = Espírito Santo; $\mathrm{GO}=$ Goiás; $\mathrm{MG}=$ Minas Gerais; $\mathrm{PR}=$ Paraná).

Figure 2. A-C. Distribution restricted to southern portion of the Espinhaço Range: A-B. Species that occur in more than one "serra". C. Endemic species of only one "serra". D. Distribution in the Espinhaço Range and adjacent areas until disjoint in the Central Plateau (BA = Bahia; ES = Espírito Santo; GO = Goiás; MG = Minas Gerais; PR = Paraná). 
Triângulo Mineiro. Chamaecrista potentilla apresenta a maior parte das ocorrências concentrada no Planalto de Diamantina, Serra do Cabral (região de Joaquim Felício) e Serra do Ambrósio (região de Rio Vermelho), mas há ocorrências em áreas adjacentes como na Serra da Canastra e outros municípios próximos como Alpinópolis e Furnas. Chamaecrista cardiostegia é conhecida de serras no centro e norte do Espinhaço mineiro, de serras no Triângulo Mineiro e da Serra dos Cristais no sudeste de Goiás, estas últimas constituintes do Arco de Brasília, e ainda com um único registro (Bortoluzzi et al. 2007) em mancha de cerrado no Paraná. O padrão disjunto Espinhaço-Goiás foi exemplificado por Giulietti \& Pirani (1988) com espécies de Diplusodon (Lythraceae), porém uma distribuição chegando a Goiás passando pela Serra da Canastra (parte do Arco de Brasília) parece ser inédita na literatura. Em relação às disjunções Canastra-Espinhaço, podemos encontrar 15 espécies de Asclepiadoideae (Apocynaceae) (Rapini et al. 2001, Farinaccio \& Mello-Silva 2004). Recentemente foram citadas algumas espécies de Velloziaceae indo do Espinhaço até serras da região da Serra da Canastra, incluindo afloramentos no nordeste de São Paulo (Mello-Silva 2005), e um padrão semelhante foi reportado também para uma ave (Polistictus superciliares) endêmica dos campos rupestres (Vasconcelos 2008). Segundo Giulietti \& Pirani (1988) a similaridade geológica física e climática dessas serras possibilita a manutenção dessas populações disjuntas, talvez remanescentes de uma maior continuidade pretérita.

3.2. Distribuição em Serras do Espinhaço e em uma área disjunta no Espírito Santo - Neste padrão está inclusa uma única espécie: C. mucronata (figura 3A), que ocorre desde o extremo sul da Cadeia do Espinhaço, é muito comum na região de Ouro Preto, não tem registros na região central da formação, volta a ser frequente no Estado da Bahia, principalmente na Chapada Diamantina, e exibindo uma distinta disjunção revelado por coletas no litoral do Espírito Santo, Município de Linhares, em solo arenoso de região quase ao nível do mar. O padrão disjunto Espinhaço-restinga é relatado para algumas espécies de Leiothrix (Eriocaulaceae), Phyllanthus (Phyllanthaceae), Xyris (Xyridaceae) e Vellozia dasypus Seub. (Velloziaceae) (Giulietti \& Pirani 1988). Recentemente, Lima \& Pirani (2003, 2007) também citaram algumas espécies de Croton (Euphorbiaceae). Segundo esses autores o padrão de distribuição disjunta campos rupestres - restingas (CR - R) seria decorrente de fatores climáticos e edáficos em comum entre essas áreas atualmente, como solos arenosos, alta insolação e frequentes períodos de grande umidade atmosférica. Flutuações climáticas pretéritas podem ter provocado retração da cobertura florestal e expansão das vegetações abertas campestres; a ausência atual das condições supracitadas nas áreas intermediárias limitaria as espécies às áreas disjuntas observadas presentemente. Considerando-se que a exposição das areias das restingas data do Quartenário e que a formação associada ao Espinhaço é muito mais antiga (Souza et al. 2005), pode-se supor uma colonização mais recente da região costeira por essas espécies. Contudo, esse padrão tem que ser examinado com cautela, pois Alves et al. (2007) mostraram que dos 56 táxons aos quais já se atribuiu tal disjunção, apenas $9(16 \%)$ o exibem efetivamente.

3.3. Distribuição centrada em serras do Espinhaço e adjacências com uma área disjunta no norte da América do Sul - Neste padrão está incluída uma única espécie: Chamaecrista roraimae (figura 3B). Trata-se da espécie mais amplamente distribuída da série, ocorrendo na Guiana e Venezuela (Monte Roraima) e no Brasil, nos Estados de Roraima, Bahia e Minas Gerais. É frequente no Estado da Bahia, principalmente nos campos rupestres, e até chega a ocupar regiões de menor altitude em pleno domínio da Caatinga. Por outro lado, há apenas duas referências para o norte do Estado de Minas Gerais, a leste e oeste dos terrenos do Espinhaço, portanto sem ocorrências no setor mineiro da formação. Esse padrão de distribuição já havia sido documentado para a espécie por Irwin \& Barneby (1982). O mesmo padrão foi depois destacado por Giulietti \& Pirani (1988), que afirmam ser observado também em quatro espécies de Xyris (Xyridaceae). Em Eriocaulaceae, esses autores relatam que Leiothrix flavescens (Bong.) Ruhl., do leste do Brasil com centro no Espinhaço, ocorre disjuntamente nas montanhas da Venezuela e do Peru. As grandes disjunções, como essas, sempre representaram questões controvertidas na Biogeografia Histórica, com disputas entre as correntes defensoras de vicariância ou de dispersão a longa distância como processos explicativos (Lomolino et al. 2006). Na última década, datações sobre filogenias moleculares em vários grupos de angiospermas têm levado a inferências de dispersão a longa distância como processo prevalecente, sobretudo nas disjunções intercontinentais, devido às idades obtidas de origem e/ou diversificação dos grupos serem mais recentes que as de grandes eventos geológicos como os relacionados à deriva continental (e.g. Givnish et al. 2004, 2011, Lomolino et al. 2006). No caso da disjunção aqui apresentada, a dispersão a longa distância também 

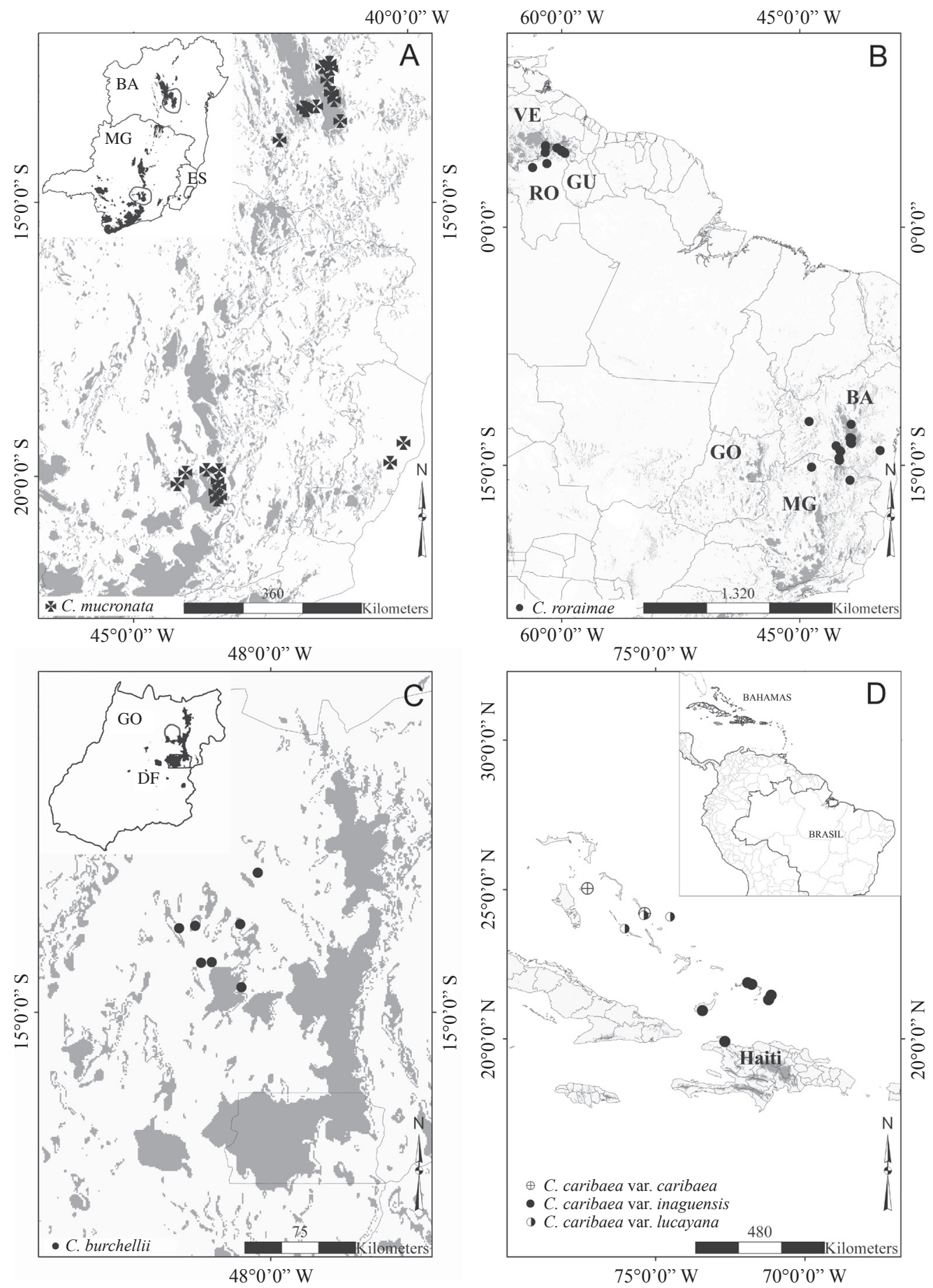

Figura 3. A-B. Distribuição na Cadeia do Espinhaço em áreas adjacentes até disjuntas: A. Distribuição em serras do Espinhaço e em área disjunta no Espírito Santo. B. Distribuição centrada em serras do Espinhaço e adjacências com uma área disjunta no norte da América do Sul. C. Distribuição restrita a outra região no Brasil. D. Distribuição restrita a outra região extrabrasileira $(\mathrm{GU}=$ Guiana; $\mathrm{RO}=$ Roraima; $\mathrm{VE}=$ Venezuela; $\mathrm{BA}=$ Bahia; $\mathrm{DF}=$ Distrito Federal; ES = Espírito Santo; $\mathrm{GO}=$ Goiás; $\mathrm{MG}=$ Minas Gerais).

Figure 3. A-B. Distribution in the Espinhaço Range and adjacent areas until disjoint: A. Distribution in the Espinhaço and a disjunct area in Espírito Santo state. B. Distribution in the Espinhaço and a disjunct area in north of South America. C. Distribution restricted to another region in Brazil. D. Distribution restricted to another region outside from Brazil $(\mathrm{GU}=$ Guiana; $\mathrm{RO}=$ Roraima; $\mathrm{VE}=$ Venezuela; $\mathrm{BA}=$ Bahia; $\mathrm{DF}=$ Distrito Federal; $\mathrm{ES}=$ Espírito Santo; $\mathrm{GO}=\mathrm{Goiás}$; $\mathrm{MG}=$ Minas Gerais). 
pode ser invocada, como nos casos de Bromeliaceae e Rapateaceae (Givnish et al. 2004, 2011, Berry \& Riina 2005). No caso da hipótese de dispersão, afinidades temporais (e.g. a longa história geológica e o isolamento em ilhas) e ambientais (e.g. baixo $\mathrm{pH}$, solos oligotróficos, alta precipitação e altas variações térmicas) entre os tepuis na Venezuela e as Serras do Espinhaço poderiam oferecer um ambiente propício ao estabelecimento de uma espécie imigrante de um outro ambiente similar. Por outro lado, os diásporos de Chamaecrista não apresentam síndrome de dispersão anemocórica ou zoocórica. Em especial neste grupo, o amadurecimento dos frutos e a torção das valvas expulsam as sementes a pequenas distâncias, um caso especial de autocoria. Isso favorece um cenário de vicariância, onde a existência pretérita de habitats abertos, não muito afastados entre si, teria sido propícia à expansão gradual de populações de Chamaecrista roraimae até o norte do Brasil, com posterior extinção nas áreas intermediárias quando novas mudanças climáticas tivessem eliminado esses habitas intermediários. No entanto, não existem ainda datações de diversificação desse grupo e as informações sobre retrações e expansões das vegetações ainda são escassas, o que dificulta a defesa de vicariância ou dispersão para justificar o evidente padrão de disjunção desta espécie.

4) Distribuição restrita a outra região no Brasil Chamaecrista burchellii (figura 3C), endêmica das serras goianas (Município de Niquelândia), é a única representante da série nessa região e morfologicamente semelhante a $C$. choriophylla, distintas principalmente pelo número de pares de folíolos e tamanho do pedicelo..

5) Distribuição restrita a uma região extrabrasileira Chamaecrista caribaea (figura 3D), com três variedades (var. caribaea, var. inaguensis e var. lucayana) consideradas espécies distintas por Britton (1917), é endêmica da região do Caribe, ocorrendo nas Bahamas e no Haiti, sendo a única espécie da série que ocorre exclusivamente próxima ao nível do mar. Quando comparamos a distribuição das variedades percebemos que a var. lucayana é comum nas ilhas do sul, var. inaguensis nas ilhas centrais e a var. caribaea na região norte. Essa espécie era considerada mais proximamente relacionada com as espécies pertecentes à série Chamaecrista por Bentham (1871). No entanto, Irwin \& Barneby (1982) circunscreveram a série Coriaceae incluindo $C$. caribaea, principalmente pelo hábito arbustivo e inflorescência exatamente axilar (e não supraaxilar, situação ocasionada por uma aderência parcial do pedicelo ao ramo, comum na série Chamaecrista). Porém, outras características de C. caribaea, como a forma dos nectários extraflorais e estrutura do sistema subterrâneo, distingue-a muito do restante das espécies da série Coriaceae. Por isso, provavelmente estudos morfológicos e filogenéticos mais detalhados deverão evidenciar que possivelmente essa espécie não pertença à série Coriaceae. Mas, caso esse posicionamento se mantenha, C. caribaea constituirá uma intrigante disjunção da série, fomentando hipóteses acerca da sua história biogeográfica e sobre sua adaptação aos fatores edáficos e climáticos onde ocorre, tão contrastantes com os da maioria das espécies do grupo.

Pode-se perceber que a maioria das espécies de Chamaecrista sect. Chamaecrista ser. Coriaceae (18 das 20 spp. e 24 dos 27 táxons) segue os padrões de distribuição já descritos para táxons componentes da vegetação da Cadeia do Espinhaço. A análise prévia de algumas espécies ( $C$. distichoclada, $C$. lagotois, C. olesiphylla, C. roraimae e C. ulmea) realizada por Giulietti \& Pirani (1988) já indicava essa situação para a série. A associação da alta riqueza específica do grupo no setor mineiro da Cadeia do Espinhaço ao fato de apenas cinco espécies do grupo se estenderem até a Chapada Diamantina, o setor norte da Cadeia, reforça a hipótese de existência de uma barreira de migração entre esses blocos, proposta por Harley (1988). Tal barreira seria constituída pelo hiato com terrenos de baixa altitude existente entre esses dois setores montanhosos, com cerca de 300 $\mathrm{km}$ de distância. Esse hiato pode ter impossibilitado a migração de muitas espécies diferenciadas nessas regiões no passado e continuar atuando desse modo sob as condições climáticas atuais. Tal barreira levaria ao isolamento geográfico e interrupção de fluxo gênico entre populações dos dois setores. Com efeito, em diferentes grupos de plantas tem sido evidenciada diferenciação genética entre populações dessas duas porções, por meio de inversões na frequência relativa de alelos e alelos exclusivos (e.g. Jesus et al. 2001, Ribeiro et al. 2007, Rapini et al. 2009), o que sugere a falta de fluxo gênico entre elas há muito tempo.

Reporta-se que ilhas isoladas e montanhas são frequentemente ricas em endemismos (Berry \& Riina 2005, Lomolino et al. 2006). Embora geralmente se invoque o isolamento de populações nas áreas elevadas como causador de especiação alopátrida, a idade dos endemismos montanos é muito controvertida, pois eles podem ser ou muito antigos ou produtos de radiação recente, com rápidas especiações. Essa última situação é referida, por exemplo, por Hughes \& Eastwood (2006) para um grande clado de Lupinus (Leguminosae) das 
montanhas dos Andes, uma vez que a orogenia andina data dos últimos 20 a 15 milhões de anos (Burnham \& Graham 1999). Por outro lado, como o conjunto orográfico do Espinhaço é muito mais antigo (Souza et al. 2005), muitos dos seus endemismos podem ter se diferenciado em épocas muito remotas.

Prance (1994) classificou os campos rupestres da Cadeia do Espinhaço como uma "fitocória em arquipélago", proposta que considera "fitocórias" as áreas dentro da qual ocorrem mais de mil espécies endêmicas. Esse autor defende que a distribuição insular dos campos rupestres facilitaria a especiação alopátrida devido ao isolamento das populações nos topos das serras, do mesmo modo que Harley (1988), Giulietti \& Pirani (1988) e Giulietti et al. (1997). Os dois casos de endemismos de Chamaecrista fora da Cadeia do Espinhaço devem ser mais explorados e hipóteses mais consistentes sobre a origem das espécies endêmicas podem surgir com a obtenção de filogenias sobre o grupo. Diversos autores (e.g. Alves \& Kolbek, 1994; Harley 1995) têm discutido as causas de surgimento e manutenção de espécies endêmicas nessas áreas montanhosas, sendo esse um tema que deve experimentar mais desenvolvimento com o avanço dos estudos filogenéticos e biogeográficos (incluindo filogeográficos) em andamento ou sendo iniciados no Espinhaço e outras áreas planaltinas brasileiras. As semelhanças e divergências morfológicas observadas entre algumas espécies e variedades de Chamaecrista também podem vir a serem melhor compreendidas com esses tipos de estudos, principalmente os filogeográficos (baseados em marcadores moleculares ou em isozimas, e.g. Jesus et al. 2001, Silva et al. 2007). Uma expressiva diversidade genética entre populações próximas parece ser comum nos campos rupestres e isso pode ser considerado estreitamente relacionado aos diversos graus de diferenças morfológicas observadas entre os muitos morfotipos e táxons de Chamaecrista dessa região.

Conceição et al. (2009) inferiram que pedicelo bibracteolado, fruto com valvas retorcidas quando maduro, antera pubescente linha de sutura e a presença de nectários extraflorais no formato côncavo são possíveis sinapomorfias de Chamaecrista. Nessa filogenia se observou que o surgimento dos tricomas glandulares foi concomitante com o desaparecimento dos nectários na seção Absus proposta por Irwin \& Barneby (1982). Segundo Conceição et al. (2009) o surgimento de tricomas glandulares pode ter garantido o sucesso do grupo nos cerrados e campos rupestres. No entanto, parece não ser o caso de $C$. sect. Chamaecrista ser. Coriaceae, que não apresenta os tricomas glandulares. Nesse clado provavelmente outras características foram importantes para o sucesso das plantas no cerrado, em especial os nectários extraflorais, folhas rígidas e coriáceas e a presença de um sistema subterrâneo bem desenvolvido, denominado xilopódio (Irwin \& Barneby 1982). A presença de uma rica rede de vascularização foliar (com diversas nervuras de calibre maior) e a presença de estípulas bem desenvolvidas protegendo totalmente as folhas jovens, também podem ser características importantes no grupo endêmico de Minas Gerais (Rando 2009). Essas características morfo-anatômicas podem constituir adaptações que, provavelmente, garantiram a sobrevivência e levaram à diversificação dessas espécies em áreas com longos períodos de estiagem e sujeitas a queimadas. Um caráter pode ser definido como uma adaptação quando é resultado de um processo de seleção natural para desempenhar uma função biológica particular e que promove um aumento no sucesso reprodutivo em relação a outros organismos que não apresentam esse caráter (Gould \& Vrba 1982). Portanto, necessita-se ainda de um arsenal maior de informações biológicas e filogenéticas sobre esse grupo, para que se possa testar adequadamente a hipótese de que as características morfológicas discutidas acima sejam efetivamente adaptativas.

A análise de distribuição geográfica de Chamaecrista ser. Coriaceae acrescenta mais dados que reforçam as evidências de alto grau de endemismo em regiões com altitude elevada dentro do domínio do Cerrado. Porém, segundo Fiaschi \& Pirani (2009) a explicação desses padrões de diversidade ainda é muito especulativa, uma vez que a maioria dos estudos não apresenta hipóteses filogenéticas sobre esses táxons endêmicos. Estudos filogenéticos, baseados em dados morfológicos e moleculares, podem trazer nova luz sobre a evolução desse grupo tão marcante do cerrado e dos campos rupestres. A determinação da idade dos clados nessas filogenias com métodos de datação molecular e/ou incremento dos raros testemunhos fósseis disponíveis no grupo, possivelmente permitirá avançar com mais segurança nesse tipo de consideração.

Agradecimentos - Os autores agradecem à Fapesp, pela bolsa de mestrado; ao New York Botanical Garden, pelo prêmio Rupert Barneby Award concedido à primeira autora; à Myndel Botanical Foundation, pelo auxílio concedido; aos curadores de herbários consultados; aos Ibama e Instituto Florestal de Minas Gerais, responsáveis pelas unidades de conservação visitadas; ao Anselmo Nogueira, pelas sugestões na redação do texto; e, principalmente, aos revisores anônimos desse artigo que realizaram uma revisão detalhada e valiosos comentários e sugestões. 


\section{Referências bibliográficas}

AB'SABER, A.N. 1967. Domínios morfoclimáticos e províncias fitogeográficas no Brasil. Orientação 3: 45-48.

ALVES, R.J.V. \& KOLBEK, J. 1994. Plant species endemism in savanna vegetation on table mountains (Campo Rupestre) in Brazil. Vegetatio 113:125-139.

ALVES, R.J.V., CARDIN, L. \& KROPF, M.S. 2007. Angiosperm disjunction "campos rupestres - restingas": a re-evaluation. Acta Botanica Brasilica 21:675-685.

BENTHAM, G. 1871. Revision of the genus Cassia. Transactions of the Linnean Society 27:503-591.

BERRY, P.E \& RIINA, R. 2005. Insights into diversity of the Pantepui Flora and the biogeographic complexity of the Guiana Shield. Biologiske Skrifter 55:145-167.

BOGGAN, J., FUNK, V., KELLOFF, C., HOFF, M., CREMERS, G. \& FEUILLET, C. 1997. Checklist of the plants of the Guianas. $2^{\text {nd }}$ ed., Smithsonian Institution, Washington, p.1-238.

BORTOLUZZI, R.L.C., MIOTTO, S.T.S. \& REIS, A. 2007. Novos registros de Chamaecrista Moench e Senna Mill. (Leguminosae - Caesalpinioideae - Cassiae) na flora sul brasileira. Iheringia 62:121-130.

BRITTON, N.L. 1917. Studies of West Indian plants - IX. Journal of the Torrey Botanical Society 44:1-37.

BURNHAM, R.J. \& GRAHAM, A. 1999. The history of neotropical vegetation: new developments and status. Annals of the Missouri Botanical Garden 86:546-589.

CAMARGO, R.A. \& MIOTTO, S.T.S. 2004. O gênero Chamaecrista Moench(Leguminosae-Caesalpinioideae) no Rio Grande do Sul. Iheringia 59:131-148.

CONCEIÇÃO, A.S., QUEIROZ, L.P. \& LEWIS, G.P. 2001. Novas espécies de Chamaecrista Moench (Leguminosae - Caesalpinioideae) da Chapada Diamantina, Bahia, Brasil. Sitientibus, série Ciências Biológicas 1:110-115.

CONCEIÇÃO, A.S., QUEIROZ, L.P., LEWIS, G.P., DE ANDRADE, M.J.G., DE ALMEIDA, P.R.M., SCHNADELBACH, A.S. \& VAN DEN BERG, C. 2009. Phylogeny of Chamaecrista (LeguminosaeCaesalpinioideae) based on nuclear and chloroplast DNA regions. Taxon 58:1168-1180.

ESRI - Environmental Systems Research Institute. 1999. ArcView GIS, version 3.2. Redlands, California.

FARINACCIO, M.A. \& MELLO-SILVA, R. 2004. Asclepiadoideae (Apocynaceae) do Parque Nacional da Serra da Canastra, Minas Gerais, Brasil. Boletim de Botânica da Universidade de São Paulo 22:53-92.

FIASCHI, P. \& PIRANI, J.R. 2008. Padrões de distribuição geográfica das espécies de Schefflera J.R. Forst. \& G. Forst. (Araliaceae) do Brasil extra-amazônico. Revista Brasileira de Botânica 31:633-644.

FIASCHI, P. \& PIRANI, J.R. 2009. Review of plant biogeographic studies in Brazil. Journal of Systematics and Evolution 47:1-20.
FRANCINO, D.M.T. 2006. Anatomia foliar de espécies de Chamaecrista Moench (Leguminosae/Caesalpinioideae) ocorrentes em campo rupestre. Dissertação de mestrado, Universidade Federal de Viçosa, Viçosa.

FUNK, V., HOLLOWELL, T., BERRY, P., KELLOFF, C. \& ALEXANDER, S.N. 2007. Checklist of the plants of the Guiana shield (Venezuela: Amazonas, Bolivar, Delta Amacuro, Guian, Surinam, French Guiana). Contributions from United States National Herbarium 55:1-584.

GIULIETTI, A.M. \& PIRANI, J.R. 1988. Patterns of geografic distribution of some plants species from the Espinhaço Range, Minas Gerais and Bahia, Brazil. In Proceedings of a Workshop on Neotropical Distribution Patterns (W.R. Heyer \& P.E. Vanzolini, eds.). Rio de Janeiro, Anais da Academia Brasileira de Ciências, p.39-69.

GIULIETTI, A.M., PIRANI, J.R. \& HARLEY. R.M. 1997. Espinhaço Range region, Eastern Brazil. In The Americas centres of plant diversity: A guide and strategy for their conservation. (S.D. Davis, V.H. Heywood, O. HerreraMacbryde, J. Villa-Lobos \& A.C. Hamilton, eds.). IUCN Publication Unity, Cambridge, v.3, p.397-404.

GIULIETTI, A.M., RAPINI, A., ANDRADE, M.J.G., QUEIROZ, L.P. \& SILVA, J.M.C. (ORG.) 2009. Plantas raras do Brasil. Conservação Internacional, Belo Horizonte.

GIVNISH, T.J., MILLAM, K.C., EVANS, T.M., HALL, J.C., PIRES, J.C., BERRY, P.E. \& SYSTSMA, K.J. 2004. Ancient vicariance or recent long-distance dispersal? Inferences about phylogeny and South America-Africa disjunctions in Rapateaceae and Bromeliaceae based on $\mathrm{ndhF}$ sequence data. International Journal of Plant Sciences 165:S35-S54.

GIVNISH, T.J., BARFUSS, M.H.G., VAN EE, B., RIINA, R., SCHULTE, K., HORRES, R, GONSISKA, P.A., JABAILY, R.S., CRAYN, D.M., SMITH, J.A.C., WINTER, K., BROWN, G.K., EVANS, T.M., HOLST, B.K., LUTHER, H., TILL, W., ZIZKA, G., BERRY, P.E. \& SYTSMA, K.J. 2011. Phylogeny, adaptive radiation, and historical biogeography in Bromeliaceae: insights from an eight-locus plastid phylogeny. American Journal of Botany 98:872-895.

GOULD, S.J. \& VRBA, E.S. 1982. Exaptation - a missing term in the science of form. Paleobiology 8:4-15.

HARLEY, R.M. 1988. Evolution and distribution of Eriope (Labiatae), and its relatives in Brazil. In Proceedings of a Workshop on Neotropical Distribution Patterns (W.R. Heyer \& P.E. Vanzolini, eds.). Rio de Janeiro, Anais da Academia Brasileira de Ciências, p.71-119.

HARLEY, R.M. 1995. Introdução. In Flora of the Pico das Almas - Chapada Diamantina, Bahia, Brasil (B.L. Stannard, ed.). Royal Botanic Gardens, Kew, p.43-78.

HUGHES, C. \& EASTWOOD, R. 2006. Island radiation on a continental scale: exceptional rates of plant diversification after uplift of the Andes. Proceedings of the National Academy of Sciences 103:10334-10339. 
IRWIN, H.S. \& BARNEBY, R.C. 1977. Monographic studies in Cassia (Leguminosae-Caesalpinioideae). IV. Supplementary Notes on Section Apoucouita Bentham. Brittonia 29:277-290.

IRWIN, H.S. \& BARNEBY, R.C. 1978. Monographic studies in Cassia (Leguminosae - Caesalpinioideae) III. Sections Absus and Grimaldia. Memoirs of the New York Botanical Garden 30:1-277.

IRWIN, H.S. \& BARNEBY, R.C. 1982. The American Cassinae: a synoptical revision of Leguminosae Tribe Cassieae subtribe Cassinae in the New World. Memoirs of the New York Botanical Garden 35:636-918.

JESUS, F.F., SOLFERINI, V.N., SEMIR, J. \& PRADO, P.I. 2001. Local genetic differentiation in Proteopsis argentea (Asteraceae), a perennial herb endemic in Brazil. Plant Systematics and Evolution 226:59-68.

JOLY, A.B. 1970. Conheça a vegetação brasileira. Edusp / Polígono, São Paulo.

JOLY, C.A., AIDAR, M.P.M., KLINK, C.A., MCGRATH, D.G., MOREIRA, A.G., MOUTINHO, P., NEPSTAD, D.C., OLIVEIRA, A.A., POTT, A., RODAL, M.J.N. \& SAMPAIO, E.V.S.B. 1999. Evolution of the Brazilian phytogeography classification systems: implications for biodiversity conservation. Ciência e Cultura 51:331-348.

LEWIS, G.P. 1987. Legumes of Bahia. Royal Botanic Gardens, Kew.

LEWIS, G.P. 2005. Tribe Cassieae. In Legumes of the World (G.P. Lewis, B. Schrire, B. Mackinder \& M. Lock, eds.). Royal Botanic Gardens, Kew.

LIMA, L.R. \& PIRANI, J.R. 2003. O gênero Croton L. (Euphorbiaceae) na Cadeia do Espinhaço, Minas Gerais, Brasil. Boletim de Botânica da Universidade de São Paulo 21:299-344.

LIMA, L.R. \& PIRANI, J.R. 2007. Revisão taxonômica de Croton sect. Lamprocroton (Müll. Arg.) Pax (Euphorbiaceae s.s.). Biota Neotropica. http://www. biotaneotropica.org.br/v8n2/en/abstract?taxonomicreview+bn01108022008 (acesso em 26/04/2010).

LOMOLINO, M.V., RIDDLE, B.R. \& BROWN, J.H. 2006. Biogeography. 3 ed. Sinauer Associates, Sunderland.

MELLO-SILVA, R. 1995. Aspectos taxonômicos, biogeográficos, morfológicos biológicos das Velloziaceae de Grão Mogol, Minas Gerais, Brasil. Boletim de Botânica da Universidade de São Paulo 14:49-79.

MELLO-SILVA, R. 2005. Velloziaceae. In Flora fanerogâmica do estado de São Paulo (M.G.L. Wanderley, G.J. Shepherd, T.S. Melhem, S.E. Martins, M. Kirizawa \& A.M. Giulietti, eds.). Fapesp, RiMa, São Paulo, v.4., p.371-376.

MORRONE, J.J. 2001. Biogeografía de América Latina y el Caribe. M\&T - Manuales e Tesis, SEA, Zaragoza, 3:148.

PRANCE, G.T. 1994. A comparison of the efficacy of higher taxa and species numbers in the assessment of biodiversity in the neotropicos. Philosophical Transactions of the Royal Society of London B 345:89-99.
QUEIROZ, L.P. DE, MELLO-SILVA, R. \& APPEZZATO, B. 2004. Subfamília Caesalpinioideae. In Flora de Grão-Mogol, Minas Gerais: Leguminosae (L.P. Queiroz, coord.) Boletim de Botânica da Universidade de São Paulo 22:213-228.

RANDO, J.G. 2009. Chamaecrista seções Apoucouita, Chamaecrista e Xerocalyx na Serra do Cipó, Minas Gerais. Dissertação de mestrado, Universidade de São Paulo, São Paulo.

RAPINI, A., MELLO-SILVA, R. \& KAWASAKI, M.L. 2001. Asclepiadoideae (Apocynaceae) da Cadeia do Espinhaço de Minas Gerais, Brasil. Boletim de Botânica da Universidade de São Paulo 19:55-169.

RAPINI, A., MELLO-SILVA, R. \& KAWASAKI, M.L. 2002. Richness and endemism in Asclepiadoideae (Apocynaceae) from the Espinhaço Range of Minas Gerais, Brazil - a conservationist view. Biodiversity and Conservation 11:1733-1746.

RAPINI, A., RIBEIRO, P.L., LAMBERT, S. \& PIRANI, J.R. 2009. A flora dos campos rupestres da Cadeia do Espinhaço. Megadiversidade 4:15-23.

RIBEIRO, P.L., BORBA, P.L., SMIDT, E.C., LAMBERT, S.M., SELBACH-SCHNADELBACH, A. \& VAN DEN BERG, C. 2007. Genetic and morphological variation in the Bulbophyllum exaltatum (Orchidaceae) complex occurring in the Brazilian "campos rupestres": implications for taxonomy and biogeography. Lankesteriana 7:97-101.

ROSS, J.L.S. 1996. Geografia do Brasil. EDUSP, São Paulo. SILVA, R.M., FERNANDES, G.W. \& LOVATO, M.B. 2007. Genetic variation in two Chamaecrista species (Leguminosae), one endangered and narrowly distributed and another widespread in the Serra do Espinhaço, Brazil. Canadian Journal of Botany 85:629-636.

SIMON, M.F. \& PROENÇA, C. 2000. Phytogeographic patterns of Mimosa (Mimosoideae, Leguminosae) in the Cerrado biome of Brazil: an indicator genus of high-altitude centers of endemism? Biological Conservation 96:279-296.

SOUZA, C.R., SUGUIO, K., SANTOS, A.M. \& OLIVEIRA, P.E. 2005. Quaternário do Brasil. Holos Editora, Ribeirão Preto.

SOUZA, V.C. \& BORTOLUZZI, R.L.C. 2010. Chamaecrista In Catálogo de espécies de plantas e fungos do Brasil. (R.C. Forzza, P.M. Leitman, A.F. Costa, A.A. Carvalho Jr., A.L. Peixoto, B.M.T. Walter, C. Bicudo, D. Zappi, D.P. Costa, E. Lleras, G. Martinelli, H.C. Lima, J. Prado, J.R. Stehmann, J.F.A. Baumgratz, J.R. Pirani, L. Sylvestre, L.C. Maia, L.G. Lohmann, L.P. Queiroz, M. Silveira, M.N. Coelho, M.C. Mamede, M.N.C. Bastos, M.P. Morim, M.R. Barbosa, M. Menezes, M. Hopkins, R. Secco, T.B. Cavalcanti, \& V.C. Souza, orgs.). Jardim Botânico do Rio de Janeiro Ministério do Meio Ambiente, Rio de Janeiro. v.2, p.1008-1021. 
THIERS, B. 2009. Index Herbariorum: A global directory of public herbaria and associated staff. New York Botanical Garden's Virtual Herbarium. http://sweetgum.nybg. org/ih/
VASCONCELOS, M.F. 2008. Mountaintop endemism in eastern Brazil: why some bird species from campos rupestres of the Espinhaço Range are not endemic to the cerrado region? Revista Brasileira de Ornitologia 16:348-362. 